\title{
Focal Boost to the Intraprostatic Tumor in External Beam Radiotherapy for Patients With Localized Prostate Cancer: Results From the FLAME Randomized Phase III Trial
}

Linda G. W. Kerkmeijer, MD, PhD ${ }^{1,2}$; Veerle H. Groen, MD ${ }^{1}$; Floris J. Pos, MD, PhD³; Karin Haustermans, MD, PhD Evelyn M. Monninkhof, PhD ${ }^{5}$; Robert Jan Smeenk, MD, PhD²; Martina Kunze-Busch, PhD²; Johannes C. J. de Boer, PhD ${ }^{1}$; Jochem van der Voort van Zijp, MD, PhD ${ }^{1}$; Marco van Vulpen, MD, PhD ${ }^{6}$; Cédric Draulans, MD, PhD ; Laura van den Bergh, MD, PhD; Sofie Isebaert, $\mathrm{PhD}^{4}$; and Uulke $\mathrm{A}$. van der Heide, $\mathrm{PhD}^{3}$

PURPOSE This study investigates whether focal boosting of the macroscopic visible tumor with external beam radiotherapy increases biochemical disease-free survival (bDFS) in patients with localized prostate cancer.

PATIENTS AND METHODS In the phase III, multicenter, randomized controlled Focal Lesion Ablative Microboost in Prostate Cancer trial, 571 patients with intermediate- and high-risk prostate cancer were enrolled between 2009 and 2015. Patients assigned to standard treatment received 77 Gy (fractions of 2.2 Gy) to the entire prostate. The focal boost arm received an additional simultaneous integrated focal boost up to 95 Gy (fractions up to $2.7 \mathrm{~Gy}$ ) to the intraprostatic lesion visible on multiparametric magnetic resonance imaging. Organ at risk constraints were prioritized over the focal boost dose. The primary end point was 5-year bDFS. Secondary end points were disease-free survival (DFS), distant metastases-free survival, prostate cancer-specific survival, overall survival, toxicity, and health-related quality of life.

RESULTS Median follow-up was 72 months. Biochemical DFS was significantly higher in the focal boost compared with the standard arm (hazard ratio $0.45,95 \% \mathrm{Cl}, 0.28$ to $0.71, P<.001$ ). At 5 -year follow-up bDFS was $92 \%$ and $85 \%$, respectively. We did not observe differences in prostate cancer-specific survival $(P=.49)$ and overall survival $(P=.50)$. The cumulative incidence of late genitourinary and Gl toxicity grade $\geq 2$ was $23 \%$ and $12 \%$ in the standard arm versus $28 \%$ and $13 \%$ in the focal boost arm, respectively. Both for late toxicity as health-related quality of life, differences were small and not statistically significant.

CONCLUSION The addition of a focal boost to the intraprostatic lesion improved bDFS for patients with localized intermediate- and high-risk prostate cancer without impacting toxicity and quality of life. The Focal Lesion Ablative Microboost in Prostate Cancer study shows that a high focal boost strategy to improve tumor control while respecting organ at risk dose constraints is effective and safe.

ASSOCIATED

CONTENT

Data Supplement

Protocol

Author affiliations and support information (if applicable) appear at the end of this article.

Accepted on December 10, 2020 and published at ascopubs.org/journal/ jco on January 20 , 2021: DOI https://doi. org/10.1200/JCO.20. 02873

*Past affiliation.

J Clin Oncol 39:787-796. ๑ 2021 by American Society of Clinical Oncology

\section{INTRODUCTION}

A dose-response relationship has been described for external beam radiotherapy (EBRT) for localized prostate cancer. Whole-gland dose escalation up to 80 Gy is considered effective and safe. ${ }^{1-4}$ Further increase in dose to the entire prostate results in higher toxicity rates. ${ }^{2,5-7}$

Local recurrences of prostate cancer following radiotherapy often originate from the primary tumor site. ${ }^{8}$ Therefore, focal boosting has been proposed to increase biochemical disease-free survival (bDFS) without increasing toxicity. ${ }^{9}$ The dose to the macroscopic visible tumor on multiparametric magnetic resonance imaging (mpMRI) is increased without increasing the dose to the organs at risk
(OAR). A systematic review identified 13 single-arm studies that assessed the efficacy and safety of a focal boost using EBRT, low-dose-rate brachytherapy (BT), or high-dose-rate BT. The pooled median 5-year bDFS of the 13 studies was $85 \%$ (range, $79 \%-100 \%)^{10}$

To test the hypothesis that focal dose escalation improves bDFS in intermediate- and high-risk prostate cancer, the Focal Lesion Ablative Microboost in Prostate Cancer (FLAME) trial was conducted, comparing EBRT of 77 Gy in 35 fractions with or without a simultaneous integrated focal boost up to 95 Gy. ${ }^{11}$ As the benefit of focal boosting was unproven, the trial was designed deliberately to avoid additional toxicity by the focal boost. Therefore, prespecified dose constraints to OAR were strictly adhered to, reducing the focal boost dose if required. 


\section{CONTEXT}

\section{Key Objective}

Is it possible to improve biochemical disease-free survival by adding a focal boost to the intraprostatic lesion to whole-gland external beam radiotherapy for patients with intermediate- and high-risk prostate cancer?

\section{Knowledge Generated}

A focal boost to the intraprostatic lesion improves biochemical disease-free survival in patients with localized intermediateand high-risk prostate cancer. By prioritizing the dose constraints of the organs at risk over the focal boost dose that could be achieved, toxicity or quality of life did not deteriorate.

\section{Relevance}

Addition of a focal boost may be considered for patients treated by external beam radiotherapy for localized intermediateand high-risk prostate cancer.

\section{PATIENTS AND METHODS}

\section{Study Design}

The multicenter, phase III, randomized controlled FLAME trial was carried out in the University Medical Center (UMC) Utrecht, the Netherlands Cancer Institute in Amsterdam, the Radboudumc in Nijmegen in the Netherlands, and the niversity Hospitals Leuven in Belgium. The research Protocol was approved by the medical ethics committee of the UMC Utrecht (NL26038.041.08) for the Netherlands and University Hospitals Leuven for Belgium (B322201110225).

The FLAME study has been registered (ClincalTrials.gov identifier: NCT01168479).

\section{Participants}

Since 2009, patients with intermediate- and high-risk localized prostate cancer were included according to the Ash criteria. ${ }^{12}$ Since the Ash criteria are no longer in use in today's practice, we used the European Association of Urology risk classification ${ }^{13}$ for further analyses.

Exclusion criteria were as follows: WHO performance score $>2$, International Prostate Symptom score $\geq 20$, evidence of lymph node involvement or distant metastases, a history of pelvic radiation, prostatectomy, transurethral resection of the prostate (TURP) less than 3 months before radiotherapy, and patients who were not able to undergo MRI. All included patients provided written informed consent.

\section{Random Assignment and Masking}

The patients were randomly assigned in a 1:1 ratio to the standard or focal boost arm. Random assignment and stratification took place by an independent trial center at the UMC Utrecht, with a minimization procedure using the center as a minimization factor, ensuring balance within each stratum and overall balance. Contrary to what was intended in the trial Protocol, hormonal therapy and TURP were not used as minimization factors. Nonetheless, post hoc stratification for hormonal therapy and TURP showed well-balanced groups (Data Supplement, online only). The
FLAME trial was blinded for patients; blinding for the investigator was not possible as the treating physicians were involved in the radiotherapy planning.

\section{Procedures}

Patients randomly assigned to the standard treatment arm received conventionally fractionated EBRT consisting of 77 Gy in 35 fractions of 2.2 Gy (equivalent dose [EQD2] 81.8 Gy, assuming an $\alpha / \beta$ ratio of 1.2 ) to the prostate. Patients assigned to the focal boost arm additionally received a simultaneous integrated focal boost up to 95 Gy to the macroscopic tumor as visible on mpMRI, resulting in 35 fractions of up to 2.7 Gy (EQD2 115.8 Gy). The boost dose was reduced, if required, to meet the OAR constraints. The treatment planning, including dose constraints and achieved dose escalation, has been described before ${ }^{11,14,15}$ (Data Supplement). The mpMRI scans were performed in the four participating centers. Although the Prostate Imaging Reporting and Data System (PI-RADS) guidelines were published after the start of the FLAME trial, the mpMRI protocols were conformed to the PI-RADS recommendations. ${ }^{16,17}$ Target volumes and the OAR were delineated on a planning computed tomography scan and planning mpMRI scan. Intraprostatic lesions were contoured as gross tumor volume (GTV) using T2-weighted, diffusion-weighted imaging and dynamic contrast-enhanced sequences. One or more GTV's could be contoured per patient. The GTV contouring has been analyzed before. ${ }^{18}$ There was no margin for clinical or planned target volume of the boost. The seminal vesicles were contoured according to clinical practice. Elective regional lymph node irradiation was not performed. Intensity-modulated radiotherapy or volumetric modulated arc therapy was applied. Gold fiducial markers were implanted for position verification during treatment. (Neo)adjuvant hormonal therapy was prescribed according to clinical practice.

\section{Outcomes}

The primary end point was 5-year bDFS, defined as the time from random assignment to biochemical recurrence. 
Biochemical recurrence was defined as the lowest prostate-specific antigen (PSA) value after treatment (PSA nadir) plus $2 \mathrm{ng} / \mathrm{mL}$, according to the Phoenix criteria. ${ }^{19}$ Secondary outcomes were prostate cancer-specific survival (PCSS), overall survival (OS), toxicity, and health-related quality of life (HRQoL). Disease-free survival (DFS) and distant metastases-free survival (DMFS) were described additionally, with failure defined as biochemical recurrence and/or evidence of recurrent disease on imaging or time to the first distant metastasis.

Genitourinary (GU) and GI toxicity were assessed using the Common Terminology Criteria for Adverse Events version 3.0. ${ }^{20}$ Toxicity was scored weekly during treatment, at 1 , 6 months, and yearly thereafter up to 10 years.

To assess HRQoL, patients filled out the European Organisation for Research and Treatment of Cancer Quality of Life Questionnaire-Prostate Cancer Module (prostatespecific) questionnaire at baseline at $1,6,12,24$, and 60 months after treatment. ${ }^{21}$ Four domains urinary symptoms, bowel symptoms, sexual activity, and sexual functioning were addressed. Sexual activity and sexual functioning are presented for patients who did not receive hormonal therapy. HRQoL scores ranged from 0 to 100 . For symptom scales (urinary and bowel) higher scores indicate more symptoms. For functioning scales (sexual activity and sexual functioning) higher scores indicate better functioning. A difference in scores of more than five points between treatment arms was considered clinically relevant.

\section{Statistical Analyses}

The FLAME trial was designed to have $80 \%$ power to detect a $10 \%$ difference in 5-year bDFS between the treatment arms (alpha 0.05, one-sided). Assuming a 5-year bDFS of $64 \%$ in the standard treatment $\mathrm{arm},{ }^{4}$ we calculated a sample size including 283 patients per arm.

Primary analyses were performed according to intention-totreat. We additionally performed a per protocol analysis.

We performed Kaplan-Meier analyses with log-rank tests up to 7 years to assess differences in bDFS, DFS, PCSS, and OS between treatment arms. Patients were censored at the date of death or last follow-up. Cox regression models were performed for bDFS, DFS, and DMFS, with adjustment for center, initial prostate-specific antigen $(\mathrm{ng} / \mathrm{mL})$, T-stage, Gleason, age (years) duration of hormonal treatment (months), and timing of hormonal treatment (neoadjuvant $v$ adjuvant). A competing risk analysis according to the Fine and Gray method was performed, with death by any cause as a competing risk. ${ }^{22}$

We created a dose-response curve with the predicted probability of biochemical and distant metastatic failure up to 7 years as a function of the near minimum (D98\%) dose to the intraprostatic lesion using logistic regression.

Late toxicity was defined as toxicity from 3 months to 5 years after the start of treatment. The differences in the cumulative incidence of late grade $\geq 2$ and late grade $\geq 3$ toxicity between treatment arms were calculated.

The observed mean HRQoL per domain over time were graphically presented. Additionally, we performed linear mixed effect models for repeated measurements to assess the impact of the focal boost on HRQoL up to 5 years after treatment, with fixed effects including random assignment and time as interaction, duration of hormonal therapy, age and baseline HRQoL, and patient ID as random effect.

Statistical analyses were performed with IBM SPSS Statistics 25, RStudio and SAS enterprise version 9.4.

\section{RESULTS}

Between November 2009 and February 2015, 571 patients with intermediate- and high-risk localized prostate cancer were randomly assigned to the standard treatment $(n=$ 287 ) or focal boost arm $(n=284)$. All patients had a potential follow-up of 5 years and median follow-up was 72 months (interquartile range 58-86). Patient and treatment characteristics were well balanced at baseline [Table 1]). Hormonal treatment was equally distributed among the study arms (65\% in both groups). The mean age was 70 years (SD 6). Six patients with low-risk disease were excluded from the analyses as they did not fulfill the inclusion criteria $(n=4$ in standard treatment and $n=2$ in the focal boost arm). Twenty-nine patients (standard treatment arm $n=10$ and focal boost arm $n=19$ ) were excluded from the per protocol analyses because they did not receive their assigned treatment (Fig 1). Eight patients (standard treatment $[n=7]$, focal boost $[n=1]$ ) were excluded from the Kaplan-Meier and Cox regression analyses because they were not followed up actively.

We observed a 5-year bDFS of $85 \%$ (38 events; $95 \% \mathrm{Cl}, 80$ to 89 ) in the standard arm and of $92 \%$ ( 21 events; $95 \% \mathrm{Cl}, 87$ to 94) in the focal boost arm, which were significantly different between groups (difference: 7\%; $95 \% \mathrm{Cl}, 4.2$ to 9.8). The Kaplan-Meier curves showed significantly improved bDFS (log-rank $P<.001$ ) and DFS (log-rank $P<.001$ ) in the focal boost arm up to 7 years (Fig 2). DMFS showed no difference (log-rank $P=.26$ ). We did not find differences in OS (log-rank $P=.50)$ and PCSS (log-rank $P=.49$ ) (Data Supplement). Adjusted Cox regression analysis showed that biochemical failures were reduced by half (hazard ratio [HR] $0.45[95 \% \mathrm{Cl}$, 0.29 to 0.71$], P<.001$ ), when comparing the focal boost with standard treatment (Table 2). These analyses showed comparable results for DFS (HR 0.48 [95\% Cl, 0.32 to 0.74], $P<$ $.001)$, whereas DMFS showed no statistically significant difference (HR 0.72 [95\% Cl, 0.43 to 1.22], $P=.22$ ). Adjusted Cox regression analysis showed an $\mathrm{HR}$ of $1.26(95 \% \mathrm{Cl}, 0.83$ to $1.92, P=.27)$ for OS and $0.69(95 \% \mathrm{Cl}, 0.27$ to $1.79, P=$ .45) for PCSS. Results of the per protocol analysis did not differ from the intention-to-treat analysis (Data Supplement). Also, a competing risk analysis showed similar results (Data Supplement). 
TABLE 1. Patient and Treatment Characteristics by Randomization Arm

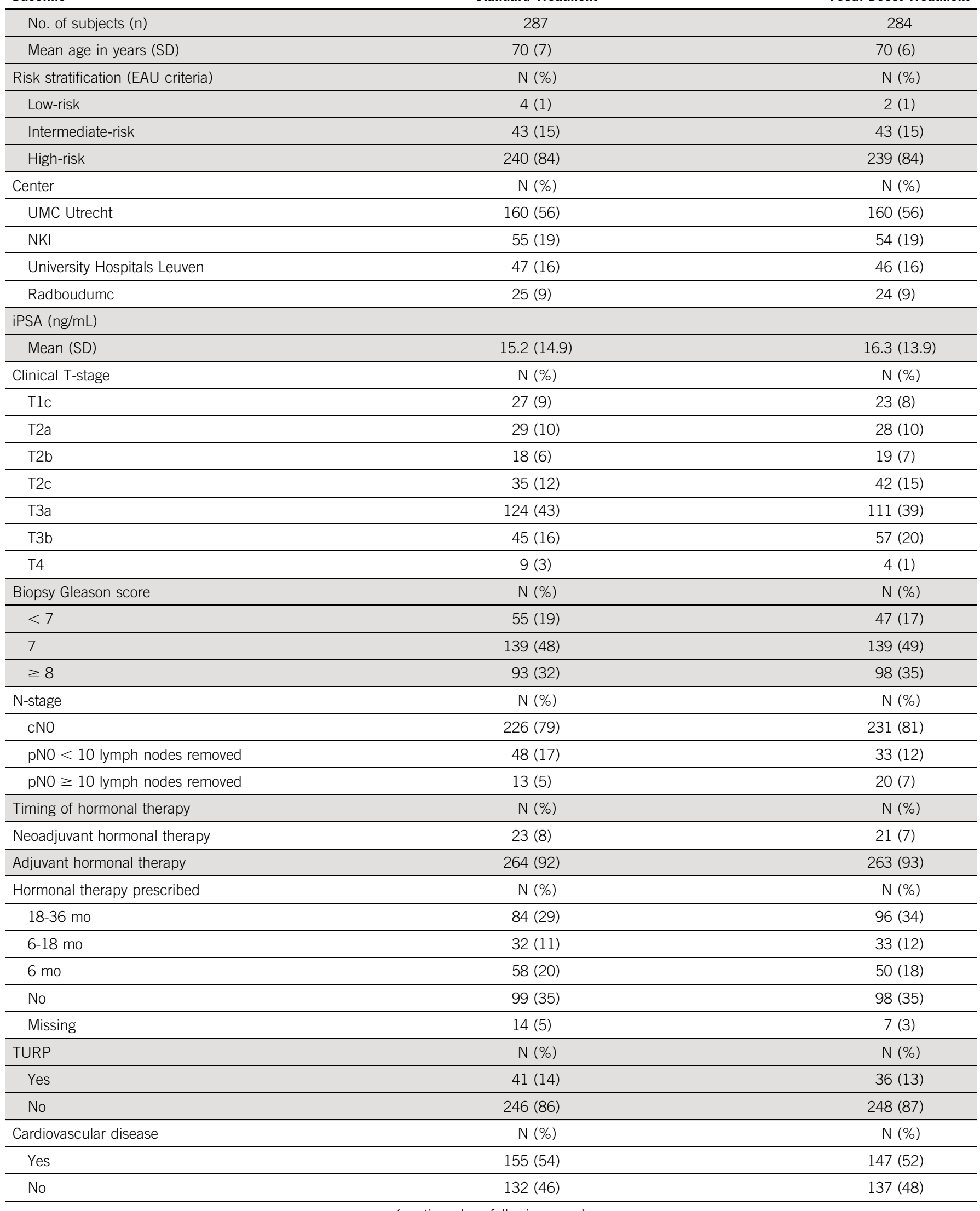


TABLE 1. Patient and Treatment Characteristics by Randomization Arm (continued)

Baseline

Standard Treatment

Focal Boost Treatment

\begin{tabular}{lrr}
\hline Diabetes mellitus & $\mathrm{N}(\%)$ & $\mathrm{N}(\%)$ \\
\hline Yes & $31(11)$ & $30(11)$ \\
\hline No & $256(89)$ & $254(89)$
\end{tabular}

Abbreviations: iPSA, initial prostate-specific antigen; TURP, trans urethral resection of the prostate.

The biochemical failure rate up to 7 years is presented as a function of achieved dose to the GTV in Figure 3. The distant metastases failure rate as a function of GTV dose is shown in the Data Supplement.

Late GU and Gl toxicity grade $\geq 2$ and grade $\geq 3$ differences were small and not statistically significant between groups. Cumulative incidences per treatment arm are presented in Table 3. One patient in the focal boost arm developed grade 4
GU toxicity, 3 years after treatment. He suffered from severe urinary incontinence for which he underwent a permanent urinary diversion. No grade $4 \mathrm{Gl}$ toxicity occurred.

The response rate was at least $85 \%$ in the first year and decreased to $55 \%$ at 5 years. We did not observe statistically significant differences in HRQOL domains between both treatmentarms (Data Supplement). The mean (95\% Cl) HRQoL per domain per time point is shown in Data Supplement.

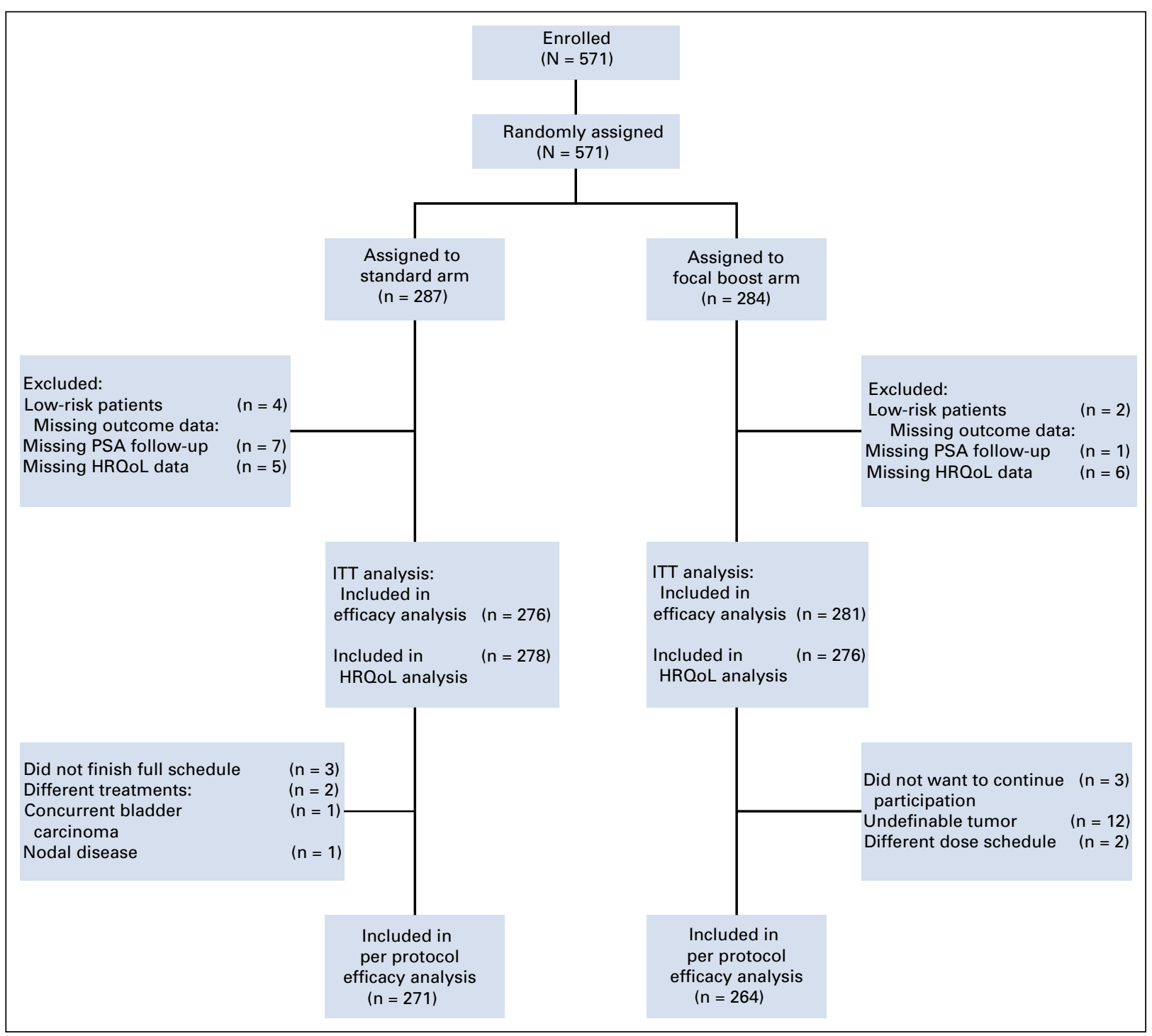

FIG 1. Trial profile. HRQoL, health-related quality of life; PSA, prostate-specific antigen. 


\section{A}

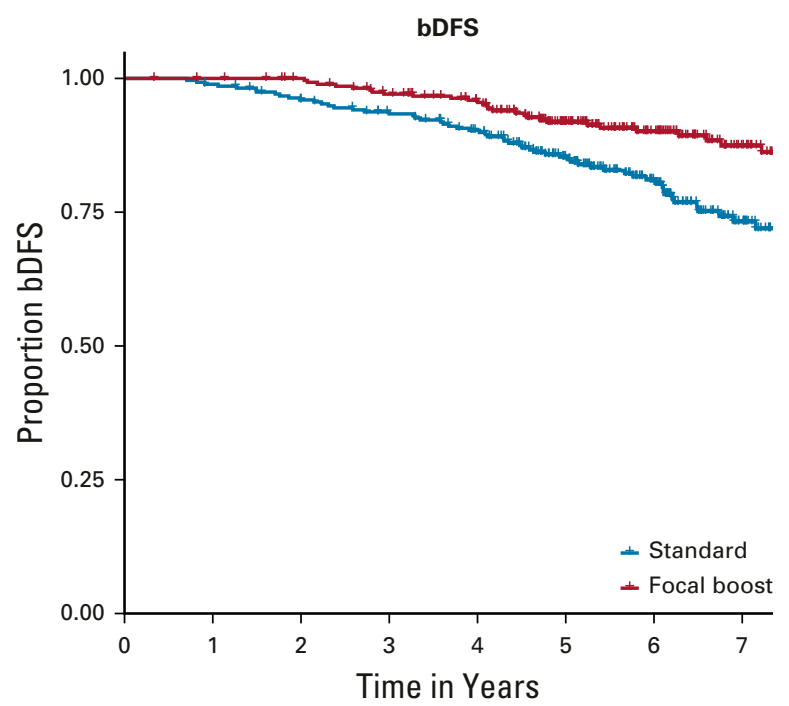

$\mathrm{N}$ at risk (cumulative events)

Standard $276(0) \quad 272(3) \quad 260(11) 247(17) 229(26) 182(38) 127(46) 67(56)$ Focal boost $281(0) \quad 279(0) \quad 274(0) \quad 261(8) \quad 244(11) \quad 188(21) \quad 135(24) \quad 80(27)$ Cumulative censoring

$\begin{array}{lllllllll}\text { Standard } & 0 & 1 & 5 & 12 & 21 & 56 & 103 & 153 \\ \text { Focal boost } & 0 & 2 & 7 & 12 & 26 & 72 & 122 & 174\end{array}$

C

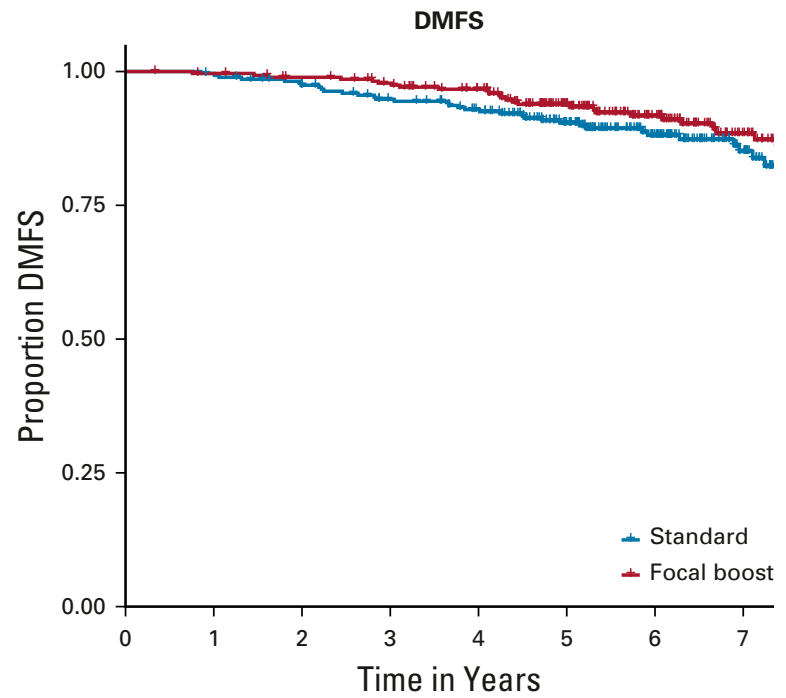

$\mathrm{N}$ at risk (cumulative events)

Standard $276(0) \quad 274(1) \quad 263(7) \quad 249(14) 235(19) \quad 192(25) \quad 133(29) \quad 73(32)$

Focal boost $281(0) \quad 278(1) \quad 272(3) \quad 264(6) \quad 247(9) \quad 194(16) \quad 140(20) \quad 84(24)$

Cumulative censoring

$\begin{array}{lllllllll}\text { Standard } & 0 & 1 & 6 & 13 & 22 & 59 & 114 & 171\end{array}$

$\begin{array}{lllllllll}\text { Focal boost } & 0 & 2 & 6 & 11 & 25 & 71 & 121 & 173\end{array}$
B

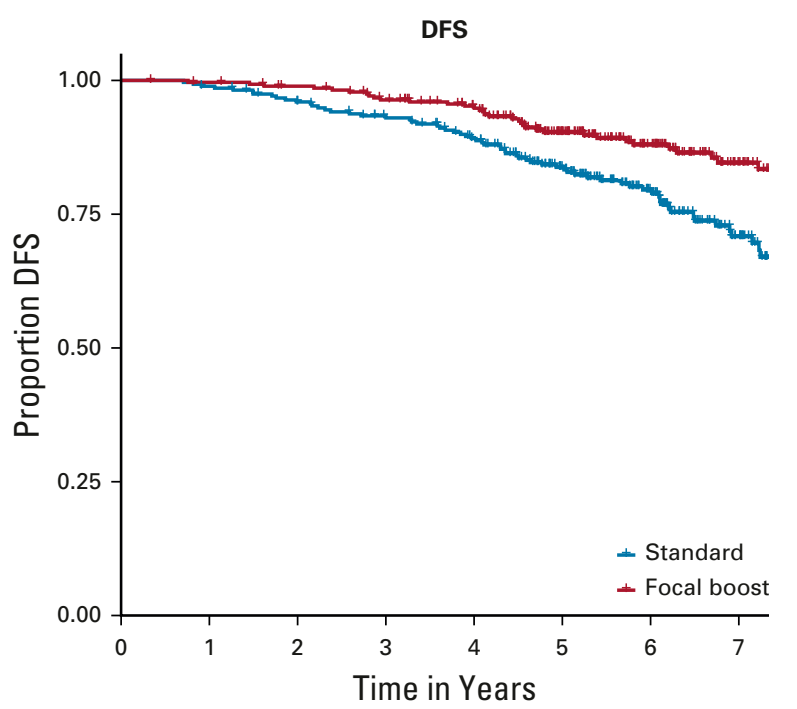

$\mathrm{N}$ at risk (cumulative events)

Standard $276(0) \quad 272(3) \quad 260(11) 246(18) 226(29) 180(42) 126(50) \quad 67(61)$ Focal boost $281(0) \quad 278(1) \quad 272(3) \quad 260(10) 243(13) 187(25) 133(29) \quad 80$ (33) Cumulative censoring

$\begin{array}{lllllllll}\text { Standard } & 0 & 1 & 5 & 12 & 21 & 54 & 100 & 148\end{array}$

FIG 2. Kaplan-Meier curves up to 7 years for (A) biochemical disease-free survival (bDFS) $(P<.001)$, (B) disease-free survival (DFS) ( $P<.001)$, and (C) distant metastases-free survival (DMFS) $(P=.26)$ comparing the standard treatment of 77 Gy in 35 fractions to the whole prostate with an additional focal boost to the macroscopic visible tumor up to 95 Gy.

Urinary HRQoL deteriorated 1 month after treatment and improved within 1 year in both treatment arms. Bowel HRQoL deteriorated less than five points from baseline in both arms and remained at a similar level during follow-up. Sexual activity in patients without hormonal therapy never deteriorated $>5$ points from baseline for both arms. 
Focal Boost Radiotherapy for Prostate Cancer in the FLAME Trial

TABLE 2. Results of Cox Regression Analysis for Biochemical Disease-Free Survival (bDFS), Disease-Free Survival (DFS) and Distant Metastases-Free Survival (DMFS) Unadjusted and Adjusted for Center, Age (y), Hormonal Treatment Duration (mo), Timing of Hormonal Treatment (Neoadjuvant $v$ Adjuvant), T-Stage, Initial PSA (ng/mL), and Gleason Score

bDFS

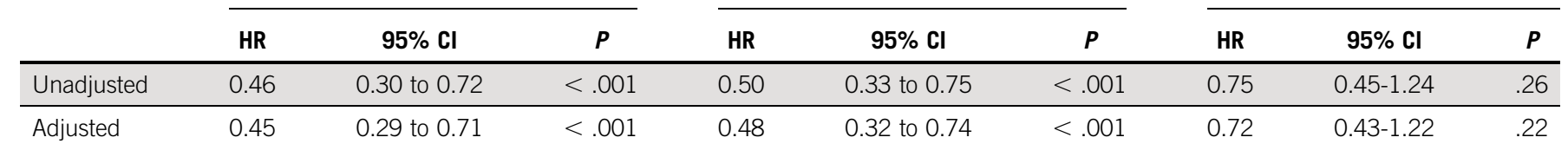

Abbreviations: HR, hazard ratio; PSA, prostate-specific antigen.

\section{DISCUSSION}

The FLAME trial is the first phase III RCT showing that the addition of a focal boost to the intraprostatic lesion(s) in EBRT for prostate cancer significantly improves 5 -year bDFS, from $85 \%$ in the standard arm to $92 \%$ in the focal boost arm. Differences in toxicity were small and not statistically significant. However, to adequately assess late GU (urethra-related) toxicity longer follow-up is required. In the FLAME trial, a urethra dose constraint was not used. However, such a constraint was incorporated in our subsequent focal boost studies. The comparable toxicity was achieved by strictly respecting dose constraints to OARs, reducing the focal boost dose if necessary. The 7\% increase in bDFS therefore likely underestimates the positive effect of focal boosting to $95 \mathrm{~Gy}$ since a clear decrease in biochemical failure rate with increasing boost dose was found. There was no significant difference in PCSS and OS. The follow-up time was relatively short to draw conclusions on survival for prostate cancer and the study was not powered for these end points. Although there was no significant difference in DMFS, the data suggest a dose-response relation for distant metastatic failure. However, a longer follow-up is required to confirm this.

In contrast to previous trials, the FLAME trial was heavily weighed to high-risk patients (84\%). Nevertheless, bDFS in the standard treatment arm was high compared with four wholegland dose-escalation RCTs including low-, intermediate-, and

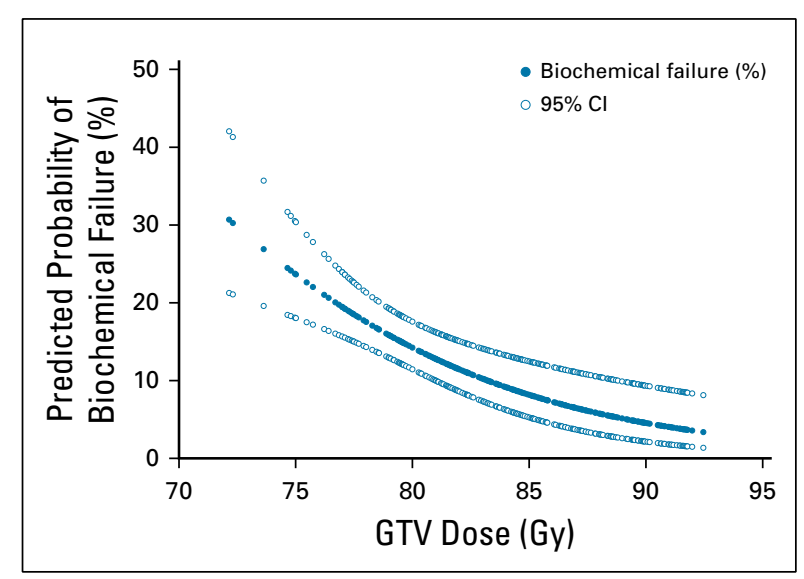

FIG 3. Predicted probability of biochemical failure up to 7 years as a function of achieved dose to the gross tumor volume (D98\%; Gy). high-risk patients, with five-year bDFS rates ranging from $64 \%$ to $76.5 \%$ in the dose-escalation arms. ${ }^{2,4,6}$ In the MD Anderson trial, a six-year freedom from failure of $70 \%$ was observed in the dose-escalation arm. ${ }^{1}$ The high overall bDFS in the FLAME trial is likely to be explained by improved disease staging before treatment. Also, the mild hypofractionation (2.2 Gy per fraction) in both arms resulted in a higher EQD2 of 81.8 Gy to the whole prostate, compared with whole-gland dose-escalation trials. ${ }^{1-4}$ As in the FLAME trial biochemical recurrence started after 1 year, this might suggest that high-risk prostate cancer is not necessarily metastasized at the time of diagnosis. The addition of (neo)adjuvant hormonal therapy may play a role in delaying biochemical failure, although 35\% of the patients in both arms did not receive hormonal therapy.

A BT boost is another strategy to increase the dose. The ASCENDE-RT (Androgen Suppression Combined with Elective Nodal and Dose Escalated Radiation Therapy) trial showed that a whole-gland low-dose rate BT boost, combined with hormonal therapy, leads to an improved bDFS compared with whole-gland EBRT. However, this came at the cost of increased 5-year grade $3 \mathrm{GU}$ toxicity. 23,24 Our results suggest that the same positive effect can be achieved without additional toxicity by limiting the boost to the intraprostatic lesion(s). In the FLAME trial, standard modern-day treatment planning techniques such as intensity-modulated radiotherapy and volumetric modulated arc therapy on conventional linear accelerators (with on-board imaging) were used. Now that the concept of focal boosting has been demonstrated to benefit bDFS, improved EBRT planning techniques ${ }^{14}$ or the use of a focal boost with BT, such as investigated in the TARGET trial (ClinicalTrials.gov identifier: NCT01802242), ${ }^{25}$ can be effective in depositing the high-boost dose without exceeding OAR constraints.

In 2009 when the FLAME trial started, a 35-39 fractions schedule was standard. Large randomized Phase III hypofractionation trials, such as the $\mathrm{CHHiP}$ (conventional or hypofractionated high dose intensity modulated radiotherapy for prostate cancer) and HYPRO (HYpofractionated irradiation for PROstate cancer) trials, ${ }^{26,27}$ showed that hypofractionated EBRT in 2.4-3.4 Gy fractions is equivalent to conventionally fractionated (1.8-2 Gy) EBRT. Around 2018 the schedule of 20 times 3Gy in 4-5 weeks (EQD2 78.8 Gy) became the new standard. ${ }^{28}$ The results of the FLAME trial also indicate that the current standard, moderately hypofractionated 20 times 3 Gy, could be improved by (focal) dose escalation. 
TABLE 3. Difference in Cumulative Incidence of Late ${ }^{a}$ Grade $\geq 2$ and Grade $\geq 3$ GU and GI toxicity

GU Toxicity

GI Toxicity

\begin{tabular}{lcccccccc} 
& 77 Gy & 95 Gy & Difference in \% (95\% CI) & $\boldsymbol{P}$ & 77 Gy & 95 Gy & Difference in \% (95\% CI) & $\boldsymbol{P}$ \\
\hline Grade $\geq 2$ & 23.0 & 27.8 & $4.8(-2.3$ to 12.0$)$ & .19 & 12.2 & 12.7 & $0.5(-5.0$ to 5.9$)$ \\
\hline Grade $\geq 3$ & 3.5 & 5.6 & $2.1(-1.3$ to 5.6$)$ & .22 & 1.4 & 1.4 & $0(-1.9$ to 2.0$)$ & .99
\end{tabular}

Abbreviation: GU, genitourinary.

a Late toxicity defined as toxicity from 3 months to 5 years after start of treatment.

A meta-analysis of randomized trials looking at doseresponse and fractionation sensitivity of prostate EBRT indicated a shallow, but highly significant, dose-response relationship. ${ }^{29}$ Although Vogelius et al. suggested saturation might be present in the dose-effect at an EQD2 of $>80 \mathrm{~Gy}$, the FLAME trial (EQD2Gy 115.8 Gy with an a/b ratio of 1.2 Gy) and ASCENDE RT trial (50 Gy + 115 Gy BT boost) proved otherwise. Dose escalation will result in better outcome; however, high doses are needed.

For low and intermediate prostate cancer the HYPO-RT-PC (HYPOfractionated RadioTherapy of intermediate risk localised Prostate Cancer) trial showed that extreme hypofractionation is noninferior to conventional fractionated radiotherapy in terms of failure-free survival and late toxicity. Acute toxicity was equal in PACE-B (intensity-modulated fractionated radiotherapy versus stereotactic body radiotherapy for prostate cancer); however, the HYPO-RT-PC showed more pronounced acute toxicity. ${ }^{30,31}$ In 2018, the American Society for Radiation Oncology (ASTRO), ASCO, and American Urological Association (AUA) Evidence-Based Guidelines stated that extreme hypofractionated $35-36,25$ Gy in five fractions may be offered to patients with low- and intermediate-risk prostate cancer. ${ }^{28}$ However, this does not apply for high risk and most likely a higher dose is needed. Several studies use focal dose escalation with a boost of 38-50 Gy (ClinicalTrials.gov identifier: NCT01409473, NCT01856855, NCT02145494, NCT01976962, NCT04245670, ISRCTN04483921, NCT02254746, NCT04045717, NCT02853110). The prospective phase II Hypo-FLAME trial investigated the safety of the technique of the FLAME trial with extreme hypofractionation. ${ }^{32}$ Mostly high-risk (75\%) patients were treated with 35 Gy in 5 weekly fractions to the whole prostate gland with an integrated boost up to 50 Gy to the mpMRI-defined tumor(s). The technique proved safe without severe acute GI or GU toxicity.

A limitation of the FLAME study was missing toxicity data, as described previously. ${ }^{15}$ We do not expect this to have influenced the late toxicity results. This is supported by an equal number of missing data in both arms. Second, the PI-RADS recommendations were published after the start of the FLAME trial and GTV contouring guidelines were not available for prostate cancer, resulting in interobserver variability. ${ }^{16,33}$ Nonetheless, we did find a significant increase in bDFS in the focal boost arm. Because of the pragmatic and practice-based approach of the trial, each center used its own OAR constraints. In addition, all centers added the same high-dose constraint for the rectum and bladder (D1cc rectum max 77 Gy and D1cc bladder max $80 \mathrm{~Gy}$ ). As the study arms were stratified for center at random assignment, center-specific constraints are not likely to have influenced the comparison of the two study arms.

An inherent limitation due to the long duration of follow-up is the change in practice over time. The FLAME trial started in 2009 and we used a pragmatic approach according to clinical practice at the time. (Neo)adjuvant hormonal therapy was given according to clinical practice. We did not collect the percent-positive biopsies and were therefore not able to calculate the University of California, San FranciscoCancer of the Prostate Risk Assessment (UCSF-CAPRA) score. $^{34}$ The International Society of Urologic Pathology ${ }^{35}$ score was implemented after patient inclusion in the FLAME trial; in the FLAME trial only the Gleason sum score was registered. Patients were staged according to clinical practice, imaging with or without a subsequent lymph node dissection. Most patients were included before a prostatespecific membrane antigen-positron emission tomography (PSMA-PET) scan was available. However, these limitations were similar in both treatment arms and we do not believe that it influenced our findings.

Strengths of our study are a large number of patients, the multicenter collaboration, and the high treatment compliance. PSA follow-up to assess the presence of bDFS was carried out thoroughly with minimal missing data, and the response rates of the HRQoL questionnaires were satisfying. Additionally, standard modern-day radiotherapy techniques were used and no additional technology or equipment was required. Thus, the implementation of a focal boost strategy does not lead to additional costs.

Although there was no survival benefit observed until now, the reduction of biochemical recurrence by $50 \%$ would probably benefit patients and their QoL. Biochemical recurrence means intensified follow-up and diagnostic examinations with associated anxiety and eventually additional treatments with subsequent toxicity, especially when this reduction in recurrence can be achieved without impacting toxicity or HRQoL compared with standard treatment and at no additional costs.

In conclusion, the FLAME trial showed that a focal boost to a high dose improves bDFS in intermediate- and high-risk 
localized prostate cancer, without additional toxicity. Focal dose escalation in (extreme) hypofractionated schedules should be further explored. As we observed a clear dose-

\section{AFFILIATIONS}

${ }^{1}$ University Medical Center Utrecht, Radiation Oncology, Utrecht, the Netherlands

${ }^{2}$ Radboud University Medical Center, Radiation Oncology, Nijmegen, the Netherlands

${ }^{3}$ The Netherlands Cancer Institute, Radiation Oncology, Amsterdam, the Netherlands

${ }^{4}$ University Hospitals Leuven, Radiation Oncology, Leuven, Belgium

${ }^{5}$ Julius Center for Health Sciences and Primary Care, University Medical Center Utrecht, Utrecht University, Utrecht, the Netherlands

${ }^{6}$ Holland Proton Therapy Center, Delft, the Netherlands

${ }^{7}$ Limburgs Oncologisch Centrum, Jessa Ziekenhuis, Radiation Oncology, Hasselt, Belgium

\section{CORRESPONDING AUTHOR}

Uulke A. van der Heide, The Netherlands Cancer Institute, Radiation Oncology, Plesmanlaan 121, Amsterdam, 1066 CX, the Netherlands, u.vd.heide@nki.nl.

\section{EQUAL CONTRIBUTION}

L.K. and V.G. share first authorship.

\section{PRIOR PRESENTATION}

Presented at the American Society for Radiation Oncology 2020 Annual Meeting, online, October 26, 2020; and ESTRO 2020, online, November 30, 2020.

\section{SUPPORT}

Supported by the Dutch Cancer Society (Grant No. KWF 10088). response relation, further improvement of tumor control may be feasible when more advanced techniques allow a higher boost dose.

\author{
CLINICAL TRIAL INFORMATION \\ NCT01168479 (FLAME)
}

\section{AUTHORS' DISCLOSURES OF POTENTIAL CONFLICTS OF INTEREST}

Disclosures provided by the authors are available with this article at DOI https://doi.org/10.1200/JC0.20.02873.

\section{DATA SHARING STATEMENT}

The datasets generated and/or analyzed during the current study are not publicly available but are available from the corresponding author on reasonable request.

\section{AUTHOR CONTRIBUTIONS}

Conception and design: Linda Kerkmeijer, Floris Pos, Karin Haustermans, Marco van Vulpen, Uulke A. van der Heide

Administrative support: Floris Pos

Provision of study materials or patients: Linda Kerkmeijer, Floris Pos, Karin Haustermans, Robert Jan Smeenk

Collection and assembly of data: Linda Kerkmeijer, Veerle Groen, Floris Pos, Karin Haustermans, Evelyn M. Monninkhof, Robert Jan Smeenk, Martina Kunze-Busch, Johannes C. de Boer, Jochem van der Voort van Zijp, Marco van Vulpen, Cédric Draulans, Laura van den Bergh, Sofie Isebaert, Uulke A. van der Heide

Data analysis and interpretation: Linda Kerkmeijer, Veerle Groen, Floris Pos, Karin Haustermans, Evelyn M. Monninkhof, Robert Jan Smeenk, Martina Kunze-Busch, Johannes C. de Boer, Cédric Draulans, Laura van den Bergh, Sofie Isebaert, Uulke A. van der Heide

Manuscript writing: All authors

Final approval of manuscript: All authors

Accountable for all aspects of the work: All authors

\section{REFERENCES}

1. Pollack A, Zagars GK, Starkschall G, et al: Prostate cancer radiation dose response: Results of the M. D. Anderson phase III randomized trial. Int J Radiat Oncol Biol Phys 53:1097-1105, 2002

2. Beckendorf $\mathrm{V}$, Guerif S, Le Prise E, et al: 70 Gy versus 80 Gy in localized prostate cancer: 5-year results of GETUG 06 randomized trial. Int J Radiat Oncol Biol Phys 80:1056-1063, 2011

3. Dearnaley DP, Jovic G, Syndikus I, et al: Escalated-dose versus control-dose conformal radiotherapy for prostate cancer: Long-term results from the MRC RT01 randomised controlled trial. Lancet Oncol 15:464-473, 2014

4. Peeters ST, Heemsbergen WD, Koper PC, et al: Dose-response in radiotherapy for localized prostate cancer: Results of the Dutch multicenter randomized phase III trial comparing 68 Gy of radiotherapy with 78 gy. J Clin Oncol 24:1990-1996, 2006

5. Kuban DA, Tucker SL, Dong L, et al: Long-term results of the M. D. Anderson randomized dose-escalation trial for prostate cancer. Int J Radiat Oncol Biol Phys 70:67-74, 2008

6. Dearnaley DP, Sydes MR, Graham JD, et al: Escalated-dose versus standard-dose conformal radiotherapy in prostate cancer: First results from the MRC RT01 randomised controlled trial. Lancet Oncol 8:475-487, 2007

7. Al-Mamgani A, van Putten WL, Heemsbergen WD, et al: Update of Dutch multicenter dose-escalation trial of radiotherapy for localized prostate cancer. Int J Radiat Oncol Biol Phys 72:980-988, 2008

8. Cellini N, Morganti AG, Mattiucci GC, et al: Analysis of intraprostatic failures in patients treated with hormonal therapy and radiotherapy: Implications for conformal therapy planning. Int J Radiat Oncol Biol Phys 53:595-599, 2002

9. Pickett B, Vigneault E, Kurhanewicz J, et al: Static field intensity modulation to treat a dominant intra-prostatic lesion to 90 Gy compared to seven field 3-dimensional radiotherapy. Int J Radiat Oncol Biol Phys 44:921-929, 1999

10. Feutren T, Herrera FG: Prostate irradiation with focal dose escalation to the intraprostatic dominant nodule: A systematic review. Prostate Int 6:75-87, 2018

11. Lips IM, van der Heide UA, Haustermans K, et al: Single blind randomized phase III trial to investigate the benefit of a focal lesion ablative microboost in prostate cancer (FLAME-trial): Study protocol for a randomized controlled trial. Trials 12:255, 2011

12. Ash D, Flynn A, Battermann J, et al: ESTRO/EAU/EORTC recommendations on permanent seed implantation for localized prostate cancer. Radiother Oncol 57: 315-321, 2000 
13. Mottet N, Bellmunt J, Bolla M, et al: EAU-ESTRO-SIOG guidelines on prostate cancer. Part 1: Screening, diagnosis, and local treatment with curative intent. Eur Urol 71:618-629, 2017

14. van Schie MA, Janssen TM, Eekhout D, et al: Knowledge-based assessment of focal dose escalation treatment plans in prostate cancer. Int J Radiat Oncol Biol Phys 108:1055-1062, 2020

15. Monninkhof EM, van Loon JWL, van Vulpen M, et al: Standard whole prostate gland radiotherapy with and without lesion boost in prostate cancer: Toxicity in the FLAME randomized controlled trial. Radiother Oncol 127:74-80, 2018

16. Weinreb JC, Barentsz JO, Choyke PL, et al: PI-RADS prostate imaging—Reporting and data system: 2015, version 2. Eur Urol 69:16-40, 2016

17. Barentsz JO, Richenberg J, Clements R, et al: ESUR prostate MR guidelines 2012. Eur Radiol 22:746-757, 2012

18. van Schie MA, Dinh CV, Houdt PJV, et al: Contouring of prostate tumors on multiparametric MRI: Evaluation of clinical delineations in a multicenter radiotherapy trial. Radiother Oncol 128:321-326, 2018

19. Roach M III, Hanks G, Thames H Jr, et al: Defining biochemical failure following radiotherapy with or without hormonal therapy in men with clinically localized prostate cancer: Recommendations of the RTOG-ASTRO Phoenix consensus conference. Int J Radiat Oncol Biol Phys 65:965-974, 2006

20. Trotti A, Colevas AD, Setser A, et al: CTCAE v3.0: Development of a comprehensive grading system for the adverse effects of cancer treatment. Semin Radiat Oncol 13:176-181, 2003

21. Borghede G, Sullivan M: Measurement of quality of life in localized prostatic cancer patients treated with radiotherapy. Development of a prostate cancerspecific module supplementing the EORTC QLQ-C30. Qual Life Res 5:212-222, 1996

22. Fine JP, Gray RJ: A proportional hazards model for the subdistribution of a competing risk. J Am Stat Assoc 94:496-509, 1999

23. Morris WJ, Tyldesley S, Rodda S, et al: Androgen suppression combined with elective nodal and dose escalated radiation therapy (the ASCENDE-RT trial): An analysis of survival endpoints for a randomized trial comparing a low-dose-rate brachytherapy boost to a dose-escalated external beam boost for high-and intermediate-risk prostate cancer. Int J Radiat Oncol Biol Phys 98:275-285, 2017

24. Rodda S, Tyldesley S, Morris WJ, et al: ASCENDE-RT: An analysis of treatment-related morbidity for a randomized trial comparing a low-dose-rate brachytherapy boost with a dose-escalated external beam boost for high- and intermediate-risk prostate cancer. Int J Radiat Oncol Biol Phys 98:286-295, 2017

25. Sanmamed N, Lee J, Berlin A, et al: Tumor-targeted dose escalation for localized prostate cancer using MR-guided HDR brachytherapy (HDR) or integrated VMAT (IB-VMAT) boost: Dosimetry, toxicity and health related quality of life. Radiotherapy and oncology. J Eur Soc Ther Radiol Oncol 149:240-245, 2020

26. Dearnaley D, Syndikus I, Mossop H, et al: Conventional versus hypofractionated high-dose intensity-modulated radiotherapy for prostate cancer: 5-year outcomes of the randomised, non-inferiority, phase 3 CHHiP trial. Lancet Oncol 17:1047-1060, 2016

27. Incrocci L, Wortel RC, Alemayehu WG, et al: Hypofractionated versus conventionally fractionated radiotherapy for patients with localised prostate cancer (HYPRO): Final efficacy results from a randomised, multicentre, open-label, phase 3 trial. Lancet Oncol 17:1061-1069, 2016

28. Morgan SC, Hoffman K, Loblaw DA, et al: Hypofractionated radiation therapy for localized prostate cancer: An ASTRO, ASCO, and AUA evidence-based guideline. J Clin Oncol 36:3411-3430, 2018

29. Vogelius IR, Bentzen SM: Dose response and fractionation sensitivity of prostate cancer after external beam radiation therapy: A meta-analysis of randomized trials. Int J Radiat Oncol Biol Phys 100:858-865, 2018

30. Brand DH, Tree AC, Ostler P, et al: Intensity-modulated fractionated radiotherapy versus stereotactic body radiotherapy for prostate cancer (PACE-B): Acute toxicity findings from an international, randomised, open-label, phase 3, non-inferiority trial. Lancet Oncol 20:1531-1543, 2019

31. Widmark A, Gunnlaugsson A, Beckman L, et al: Ultra-hypofractionated versus conventionally fractionated radiotherapy for prostate cancer: 5-year outcomes of the HYPO-RT-PC randomised, non-inferiority, phase 3 trial. Lancet 394:385-395, 2019

32. Draulans $\mathrm{C}$, van der Heide UA, Haustermans $\mathrm{K}$, et al: Primary endpoint analysis of the multicentre phase II hypo-FLAME trial for intermediate and high risk prostate cancer. Radiother Oncol 147:92-98, 2020

33. Westphalen AC, McCulloch CE, Anaokar JM, et al: Variability of the positive predictive value of PI-RADS for prostate MRI across 26 centers: Experience of the society of abdominal radiology prostate cancer disease-focused panel. Radiology 296:76-84, 2020

34. Cooperberg MR, Hilton JF, Carroll PR: The CAPRA-S score: A straightforward tool for improved prediction of outcomes after radical prostatectomy. Cancer 117 : 5039-5046, 2011

35. Gordetsky J, Epstein J: Grading of prostatic adenocarcinoma: Current state and prognostic implications. Diagn Pathol 11:25, 2016 
AUTHORS' DISCLOSURES OF POTENTIAL CONFLICTS OF INTEREST

Focal Boost to the Intraprostatic Tumor in External Beam Radiotherapy for Patients With Localized Prostate Cancer: Results From the FLAME Randomized Phase III Trial

The following represents disclosure information provided by authors of this manuscript. All relationships are considered compensated unless otherwise noted. Relationships are self-held unless noted. I = Immediate Family Member, Inst = My Institution. Relationships may not relate to the subject matter of this manuscript. For more information about ASCO's conflict of interest policy, please refer to www.asco.org/rwc or ascopubs.org/jco/authors/author-center.

Open Payments is a public database containing information reported by companies about payments made to US-licensed physicians (Open Payments).

Martina Kunze-Busch

Employment: Philips Healthcare

Stock and Other Ownership Interests: Philips Healthcare, Elekta

Jochem van der Voort van Zijp

Research Funding: Philips Healthcare, Elekta
Uulke A. van der Heide

Research Funding: Elekta

No other potential conflicts of interest were reported. 